\title{
Contribution of fortified foods to nutrient intakes in Irish teenagers aged 13 to 17 years
}

\author{
E. Walsh, J. Walton, E. Hayes, E. M. Hannon and J. A. Flynn \\ School of Food and Nutritional Science, University College Cork, Cork, Republic of Ireland
}

The objective of this study was to investigate the contribution of fortified foods to the nutrient intakes of Irish teenagers using data from the National Teens Food Survey (http://www.iuna.net). Of the 441 participants in the survey, $90 \%$ recorded consumption of one or more fortified foods in the $7 \mathrm{~d}$ food diary. Fortified foods ( $n$ 229/13\% of the 1761 food consumed) were identified by having one or more micronutrients in the ingredient list on the label of the food and contribute on an average $7 \%$ to the mean daily energy intakes.

The main food groups containing fortified foods were ready to eat breakfast cereals $(n 81)$, squashes and fruit juice drinks $(n 41)$, cereal bars ( $n$ 29), soups and miscellaneous foods (e.g. Bovril) $(n$ 17), fruit juices $(n$ 15) and yogurts and drinking yogurts $(n$ 13). The nutrients most commonly added to foods and $\%$ of foods fortified with each nutrient were: vitamin $\mathrm{B}_{6}(73 \%)$, vitamin $\mathrm{B}_{1}(69 \%)$, niacin $(68 \%)$, folic acid $(61 \%)$, vitamin $\mathrm{B}_{2}(60 \%)$, vitamin $\mathrm{B}_{12}(59 \%)$, Fe $(48 \%)$, pantothenic acid $(40 \%)$, vitamin $\mathrm{C}(31 \%)$, vitamin $\mathrm{D}(24 \%)$ and vitamin $\mathrm{E}$ and $\mathrm{Ca}(22 \%)$. The contribution of fortified foods to mean daily macro- and micronutrient intakes are reported as $\%$ of total intakes.

\begin{tabular}{lclc}
\hline Nutrient & \% of total intake & Nutrient & \% of total intake \\
\hline Total energy & 7 & $\mathrm{Fe}$ & 23 \\
Total carbohydrate & 10 & $\mathrm{Ca}$ & 7 \\
Starch & 12 & $\mathrm{Na}$ & 8 \\
Total sugars & 8 & Folate & 25 \\
Non-starch polysaccharides & 9 & Vitamin $\mathrm{B}_{1}$ & 21 \\
Fat & 2 & Vitamin B & 21 \\
Saturated fat & 1 & Vitamin B & 21 \\
Mono-unsaturated fat & 2 & Niacin & 14 \\
Poly-unsaturated fat & 5 & Vitamin D & 13 \\
Protein & 4 & Vitamin C & 6 \\
\hline
\end{tabular}

Compared to their contribution to total energy (7\%), fortified foods contribute more to intake of total carbohydrate, starch and total sugars and less to protein, total fat, saturated fat, mono-unsaturated fat and poly-unsaturated fat. The contribution of fortified foods to Na intake $(8 \%)$ is not disproportionate to their contribution to energy, considering that the estimate of Na intake does not include Na added to foods during cooking and at table. Fortified foods make an important contribution (13-25\%) to the intakes of many micronutrients, particularly $\mathrm{Fe}$, folate vitamins $\mathrm{B}_{1}, \mathrm{~B}_{2}, \mathrm{~B}_{6}$, niacin and vitamin $\mathrm{D}$.

The project was funded by the Irish Government under the National Development Plan 2000-2006. 